\title{
Vector diffraction theory of light propagation through nanostructures
}

\author{
Glen D. Gillen ${ }^{*}$ and Shekhar Guha \\ Air Force Research Laboratory, Materials and Manufacturing Directorate, Wright-Patterson Air \\ Force Base, Dayton, Ohio 45433
}

\begin{abstract}
It is well known that "vector" diffraction theory needs to be invoked to describe the propagation of light through apertures having dimensions on the order of the wavelength of light. For regions close to the aperture, use of Kirchhoff boundary conditions in the aperture plane is invalid. The Hertz vector formalism provides a way to describe the diffraction of light beams through apertures having sizes ranging from half the wavelength of light to larger values. Here we will present a summary of the method used to calculate the distribution of all of the electromagnetic field components and a Poynting vector component at and near the plane of a single elliptical aperture.
\end{abstract}

Keywords: vector diffraction theory, Hertz vector diffraction theory, near-field beam propagation, aperture effects

\section{INTRODUCTION}

Vector diffraction theory needs to be invoked for light propagation in and around structures having dimensions on the same order of magnitude as the wavelength of the incident light, as these regions are excluded from the regions of validity of approximations and assumptions invoked for the use of scalar diffraction theory. Recently vector diffraction theory has been used in a wide variety of scientific and engineering fields including focusing of light by micro-lenses [1], lithography [2], tight laser beam focusing [3], etc. For a plane wave incident upon an aperture of arbitrary shape, the Hertz vector method provides a convenient way to describe the distribution of all the components of the electromagnetic field beyond the aperture $[4,5]$. The fields can be expressed in terms of single integrals both in the geometrical "illuminated" region beyond the aperture as well as in the geometrical "shadow" region. In the boundary region between the illuminated and the shadow region it is computationally more efficient to use the two-dimensional integral expressions for the fields. In reference [4], the detailed expressions for these integrals have been presented for the case of a circular aperture in an opaque screen. Evaluation of the two-dimensional profiles of the fields as well as that of the Poynting vector in the plane of the circular aperture and for points near the aperture show strong anisotropy and interesting maxima and minima of light distributions. Strong radial asymmetries in the calculated electromagnetic fields in the aperture plane [4] agree with previously measured experimental results [6].

In this paper, the theory is extended to the case of a plane wave being diffracted by an elliptical aperture in a plane opaque conducting screen. Two additional parameters that arise over the circular aperture case are the ellipticity $(\varepsilon)$ of the aperture and the angle $(\theta)$ between the major axis of the ellipse and the polarization direction of the incident light. Using the formalism of Ref. [4] the ellipticity of the aperture is incorporated into the integration over the aperture plane. The effects of the polarization orientation of the incident light is then incorporated as the superposition of two incident orthogonal waves of appropriate amplitudes that are parallel to the ellipse's natural axes. Then the computationally efficient single integral expressions for the geometrically illuminated and the geometrical shadow regions are provided. Finally, detailed two dimensional beam profiles at the plane of the elliptical aperture as well as in the near-field region are plotted for various values of the parameters. The dependence of the on-axis intensity on the distance from the aperture is also shown.

*Also at: Anteon Corporation, 5100 Springfield Pike Suite 509, Dayton, Ohio, 45431

Nanoengineering: Fabrication, Properties, Optics, and Devices II, edited by Elizabeth A. Dobisz, Louay A. Eldada, Proc. of SPIE Vol. 5931, 59310W, (2005) · 0277-786X/05/\$15 · doi: 10.1117/12.618798 


\section{THEORY}

According to Hertz [7] all six of the components of the electromagnetic field can be derived from a single vector function I. Substitution of this vector potential into Maxwell's equations leads to expressions for the electric field,

$$
E=k^{2} \Pi+\nabla(\nabla \cdot \Pi),
$$

and the magnetic field,

$$
H=-\frac{k^{2}}{i \omega \mu_{o}} \nabla \times \Pi
$$

where $\Pi$ is the Hertz vector, $k$ is the wave number, $\omega$ is the optical frequency and $\mu_{o}$ is the permeability of free space. Bekefi [8] has shown that for diffraction of light by an aperture in a plane, the electric and magnetic fields can be calculated using just the component of the Hertz vector parallel to the incident laser polarization, which can be written as [8]

$$
\Pi_{x}(x, y, z)=\frac{i E_{o}}{2 \pi k} \iint \frac{e^{-i k \rho}}{\rho} d x^{\prime} d y^{\prime},
$$

where $x, y$, and $z$ are the coordinates of the point of interest, $E_{o}$ is the incident field amplitude, $\rho$ is the distance from the source point (primed coordinates) to the field point (unprimed coordinates), or

$$
\rho\left(x, y, z, x^{\prime}, y^{\prime}, 0\right)=\sqrt{\left(x-x^{\prime}\right)^{2}+\left(y-y^{\prime}\right)^{2}+z^{2}} .
$$

It is assumed here that the aperture plane resides at $z^{\prime}=0$, the incident light field is a plane wave, and the time dependence of the fields is $e^{-i \omega t}$. The integration of Eq. (3) is performed over the open area of the elliptical aperture in the aperture plane.

Although the electric and magnetic fields calculated using Eqs. (1) and (2) are valid for all points within the aperture plane and beyond, the double integration can become computationally time-intensive for points near the aperture plane (small values of $\rho$ ) or for repeated calculations for a large number of points of interest. By a coordinate transformation, the Cartesian double integral in Eq. (3) can be reduced to a single integral $[9,10]$, and the $x$-component of the Hertz vector at a field point lying within the geometrical illuminated region $\left(x^{2}+y^{2}<\mathrm{a}^{2}\right)$ can now be expressed as

$$
\Pi_{x}\left(x_{1}, y_{1}, z_{1}\right)=\frac{E_{o} a^{2}}{p_{1}^{2}}\left[\exp \left(-i p_{1}^{2} z_{1}\right)-\frac{1}{2 \pi} \int_{0}^{2 \pi} \exp \left(-i p_{1} q\right) d \varphi\right],
$$

where $a$ is the minor axis, $p_{1}$ is a dimensionless parameter, $\rho_{l}=2 \pi a / \lambda$, and $x, y$, and $z$ are the normalized coordinates:

$$
x_{1}=\frac{x}{a}, \quad y_{1}=\frac{y}{a}, \quad z_{1}=\frac{z}{z_{o}},
$$

where $z_{o}=2 \pi a^{2} / \lambda$. The angle $\phi$ is the angle between the $x$-axis and the line connecting $\left(x_{1}, y_{1}, 0\right)$ to the integration element along the rim of the aperture. The quantity $q$ in Eq. (5) is the distance from the field point to the rim of the aperture, or

$$
q^{2}=u^{2}+p_{1}^{2} z_{1}^{2}
$$

where $u$ is the distance from the projection of the point of interest onto the aperture plane, $\left(x_{1}, y_{1}, 0\right)$, to the elemental portion of the rim of the aperture, or

$$
u=\frac{-\left(2 x_{1} \cos \phi+2 \varepsilon^{2} y_{1} \sin \phi\right)+\sqrt{4\left(x_{1} \cos \phi+\varepsilon^{2} y_{1} \sin \phi\right)^{2}-4\left(x_{1}^{2}+\varepsilon^{2} y_{1}^{2}-\varepsilon^{2}\right)\left(\cos ^{2} \phi+\varepsilon^{2} \sin ^{2} \phi\right)}}{2\left(\cos ^{2} \phi+\varepsilon^{2} \sin ^{2} \phi\right)} \text {. }
$$

Similarly, for field points outside the geometrically illuminated region, the double integral of Eq. 3 can be expressed as a single line integral around the rim of the aperture as outlined by Carter and Williams [10], and the single integral Hertz vector for points in the geometrical shadow region can be written as 


$$
\Pi_{x}\left(x_{1}, y_{1}, z_{1}\right)=-\frac{E_{o} a^{2}}{2 \pi p_{1}^{2}} \int_{\phi_{A}}^{\phi_{B}}\left[\exp \left(-i p_{1} q_{\max }\right)-\exp \left(-i p_{1} q_{\min }\right)\right] d \phi,
$$

where $\varphi_{\mathrm{A}}$ and $\varphi_{\mathrm{B}}$ are the angles from $\left(x_{1}, y_{1}, 0\right)$ to the tangents of the ellipse, and $q_{\max }$ and $q_{\min }$ are the distances to the far and the near side of the ellipse, respectively, from $\left(x_{1}, y_{1}, 0\right)$.

The treatment above is for the special case of the polarization of the incident plane wave parallel to the major axis. If the incident laser polarization is oriented at some angle, $\theta$, with respect to the major axis, then the problem is decoupled into the superposition of two orthogonal incident light fields parallel to the $x$ and $y$-axes. One calculation is performed using the treatment above for the component of the incident light field parallel to the major axis, and the other for the component of the incident light field parallel to the minor axis. For the component parallel to the minor axis, the equations outlined above can be used if the parameters of the ellipse undergo the transformations:

$$
p_{1}^{\prime \prime}=\varepsilon p_{1}, \quad x_{1}^{\prime \prime}=-\frac{x_{1}}{\varepsilon}, \quad y_{1}^{\prime \prime}=\frac{y_{1}}{\varepsilon}, \quad z_{1}^{\prime \prime}=\frac{z_{1}}{\varepsilon^{2}}, \quad \varepsilon^{\prime \prime}=\frac{1}{\varepsilon} .
$$

After the calculations for the orthogonal fields for each component of the incident wave, the final fields are simply the superposition of the two computed orthogonal components.

\section{RESULTS}

The electric and magnetic field components are calculated from the expressions for the Hertz vector component given in Eqs. (8) and (9) by evaluating the derivatives given in Eqs. (1) and (2). The field intensity is given by the $z$-component of the Poynting vector, or

$$
S_{z}=\operatorname{Re}\left(E_{x} H_{y}^{*}-H_{x}^{*} E_{y}\right) .
$$

Figure 1(a) is a high-resolution intensity beam profile, in the aperture plane, for the diffraction of a plane wave incident upon an elliptical aperture with a minor axis equal to the half of the wavelength of the light and an ellipticity of 2 , and the incident polarization parallel to the major axis of the ellipse. Figure 1(c) is the corresponding contour plot. The plots in Fig. 1 are calculations of the $z$-component of the Poynting vector. For apertures with dimensions less than the wavelength of light, no interference maxima or minima due to the presence of the aperture are observed in the aperture plane. Only the smooth "leakage" of light through the middle and tapering off towards the edges is observed. Once the aperture dimensions become equal to or larger than the wavelength of light, the perturbation of the light field due to the presence of the aperture becomes the dominant characteristic of the intensity beam profile as illustrated in Fig. 1(b), and the corresponding contour plot Fig. 1(d), for an elliptical aperture with the minor axis equal to the wavelength of light and the major axis equal to twice the wavelength of the light.

Figure 2, similar to Fig. 1, illustrates two-dimensional intensity profiles and their corresponding contour plots for minor axes equal to 2.5 and 5 times the wavelength of the incident light, and the incident polarization parallel to the major axis of the ellipse. Along the minor axis, there are $a / \lambda$ oscillations in the intensity. Oscillations in the radial direction perpendicular to the incident polarization were also reported in Ref. [4] for a circular aperture. When contrasting diffraction patterns observed for diffraction of light by a circular aperture with the diffraction of light by an elliptical aperture, there are a few of noticeable differences between intensity beam profiles. First, for the circular case the radial oscillations were observed along the axis perpendicular to the incident polarization angle, and nearly absent (save for a variation right in the middle) along the axis of polarization, (see Figs. 1, 5-8 of Ref. [4]). The radial variations in intensity observed in the circular case followed the angular curve of the aperture edge. Contrary to the circular aperture diffraction patterns, the elliptical aperture patterns in the aperture plane still exhibit radial intensity fluctuation along the direction of the polarization of the incident plane wave. In addition, the maxima and minima observed do not strictly follow the angular curve of the rim of the aperture, but rather tend to follow a linear pattern parallel to the incident polarization angle. This tendency of the intensity maximum and minimum to follow the axis of polarization towards the middle of the ellipse is clearly evident in the contour plots of Fig. 2. 

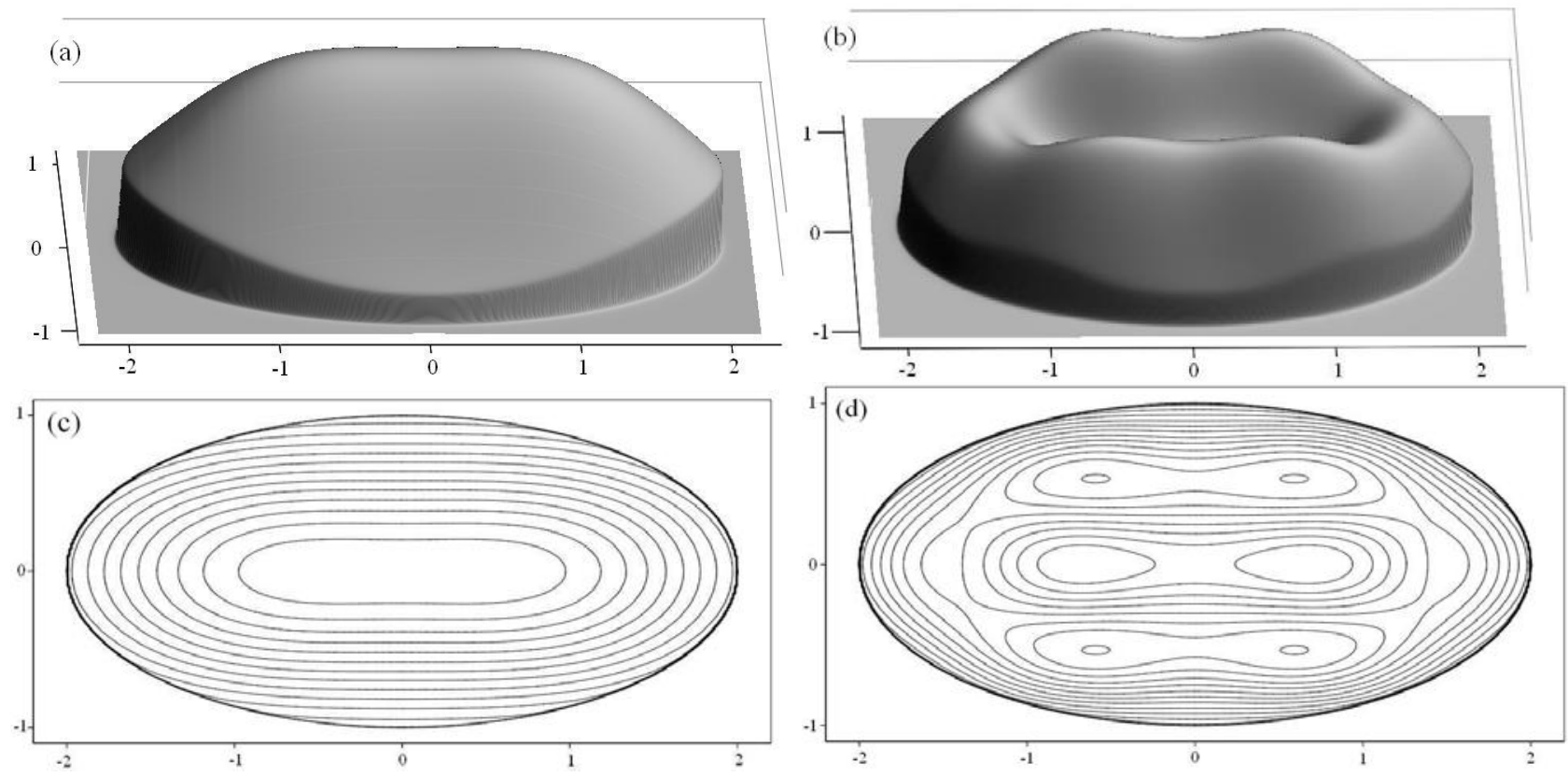

Fig. 1. Intensity distributions of the normalized z-component of the Poynting vector in the aperture plane for (a) $a=\lambda / 2$ and $b=\lambda$, (b) $a=\lambda$ and $b=2 \lambda$, and their corresponding contour plots, (c) and (d), respectively, where $a$ is the minor axis, $b$ is the major axis, and the polarization of the incident light is aligned with the major axis.
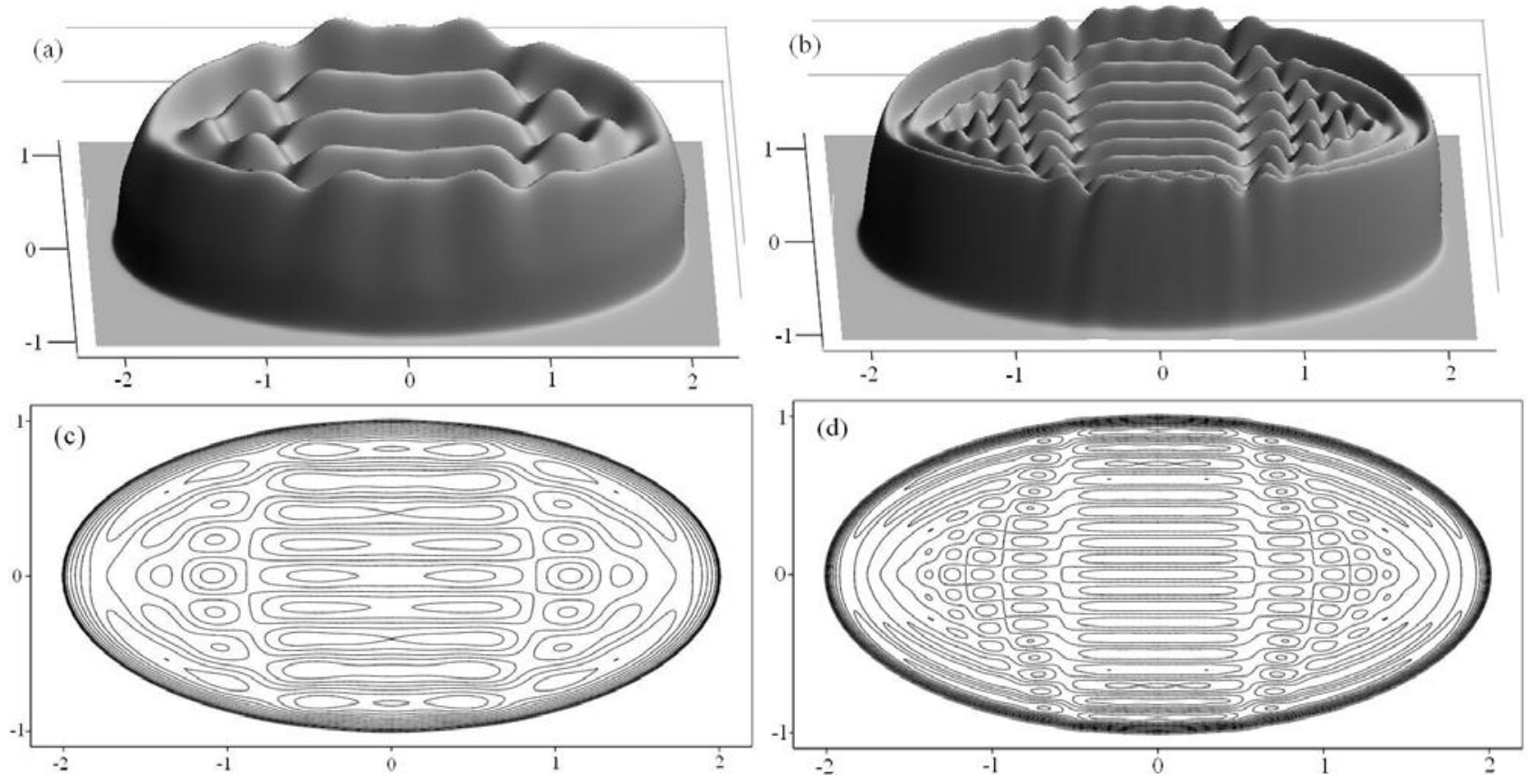

Fig. 2. Intensity distributions of the normalized z-component of the Poynting vector in the aperture plane for (a) $a=2.5 \lambda$ and $b=5 \lambda$, (b) $a=5 \lambda$ and $b=10 \lambda$, and their corresponding contour plots, (c) and (d), respectively, where $a$ is the minor axis, $b$ is the major axis, and the polarization of the incident light is aligned with the major axis. 
Figure 3 shows the effects of an initial polarization (which is not aligned with the major axis) on the calculated diffraction patterns. Figures 3(a) and (c) are surface and contour plots of the $z$-component of the Poynting vector for light incident upon the elliptical aperture with a polarization that has an angle of $\pi / 4$ with respect to the major axis. The regions within the aperture near the areas where the rim is parallel to the incident polarization show strong intensity modulations, whereas regions near portions of the rim which are perpendicular to the incident polarization show weak intensity modulations, indicating that scattering of the incident field due to the effects of the aperture rim are a significant contributor to the presence of the observed intensity modulations within the aperture. Figures 3(b) and (d) are surface and contour plots for the diffraction of light incident upon the aperture with the initial polarization aligned with the minor axis. Throughout the middle, the intensity modulations tend to align themselves parallel to the incident polarization. The persistent alignment of the intensity modulations with the incident polarization indicate that the light scattered off of the aperture rim is interfering with the incident field as well as scattered waves from other points along the rim.
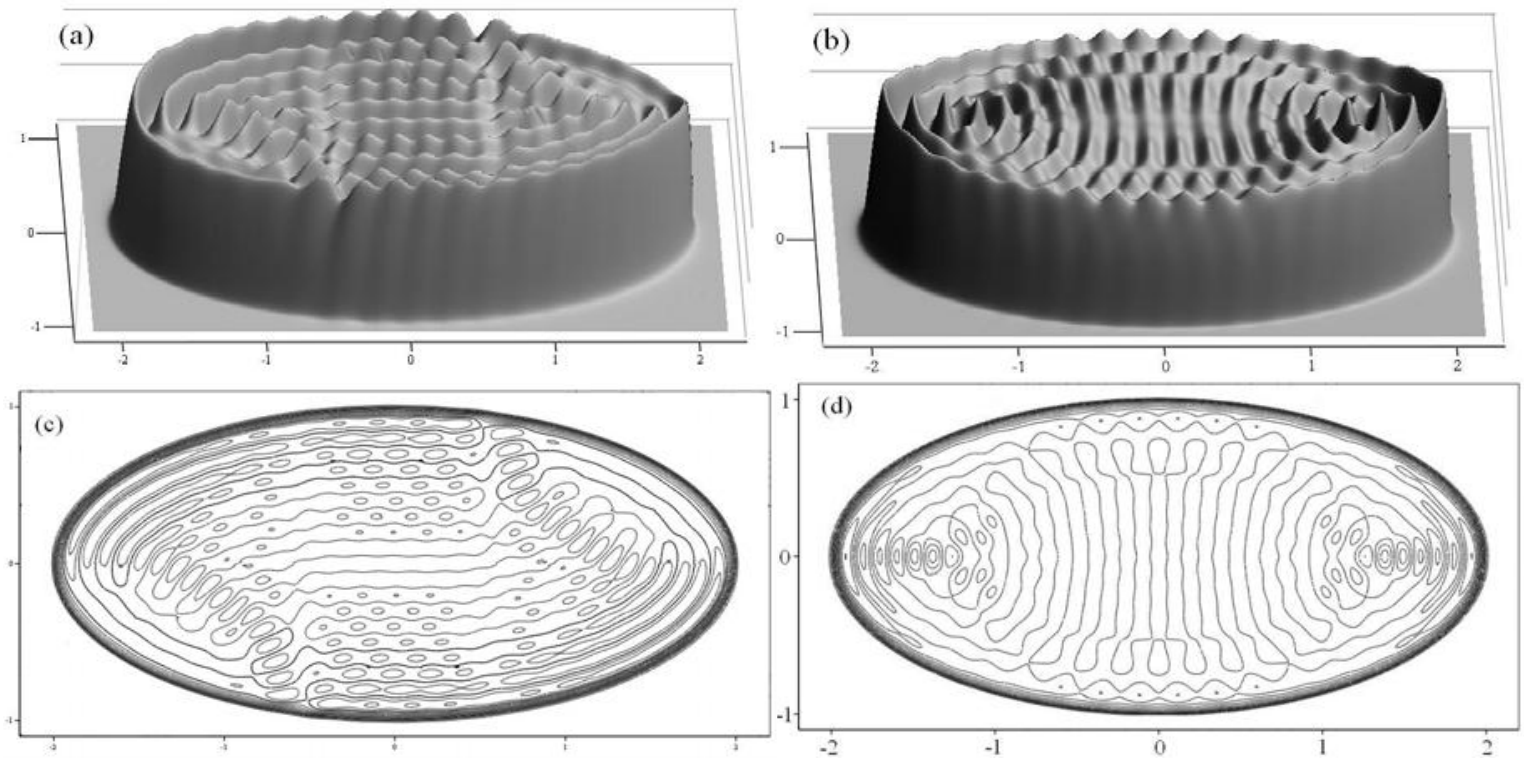

Fig. 3. Intensity distributions of the normalized z-component of the Poynting vector in the aperture plane for $a=5 \lambda$ and $b=10 \lambda$, with the incident polarization at an angle of (a) $\pi / 4$ with respect to the major axis, (b) aligned with the minor axis, and their corresponding contour plots, (c) and (d), respectively.

The plots in Fig. 4 investigate on-axis and radial intensity distributions farther from the aperture plane for an elliptical aperture with an ellipticity of 2 and a minor axis equal to 5 times the wavelength of the incident plane wave. Figure 4(a) is a plot of the on-axis value of the $z$-component of the Poynting vector. Overall, the on-axis distribution resembles what would be expected for the diffraction of light by a circular aperture with a radius equal to 5 times the wavelength of the incident plane wave (see Ref. [4]) where there would be 5 oscillations in the calculated on-axis intensity. The deviation from the expected on-axis intensity distribution for a circular aperture for the elliptical aperture of Fig. 4, mainly occurs in the range of approximately $0.07<z_{1}<1$. For a circular aperture, the last (single and smooth) on-axis maximum will occur for $z_{1}=1 /(2 \pi)$, or 0.16 . For the ellipse (with an ellipticity of 2 ) there are 8 changes in the sign of the second derivative of the on-axis intensity through this axial range, where there would be only 3 for a circular aperture. Figure 4(b) is a high-resolution intensity profile for a plane located at $z_{l}=0.054$, or the location of the fourth maximum from the aperture. Figure 4(c) is an intensity profile for the on-axis minimum located at $z_{1}=0.09$, and Fig. $4(\mathrm{~d})$ is for the plane located at $z_{l}=0.45$, or the last on-axis maximum. Strong radial asymmetries and localized field spikes exist throughout the near-field region $\left(z_{1}<1\right)$ for diffraction by elliptical apertures. Beyond an axial distance of $z_{1}$ $=1$, the intensity distributions radially smooth out with a central maximum surrounded by subtle ripples or wings. 

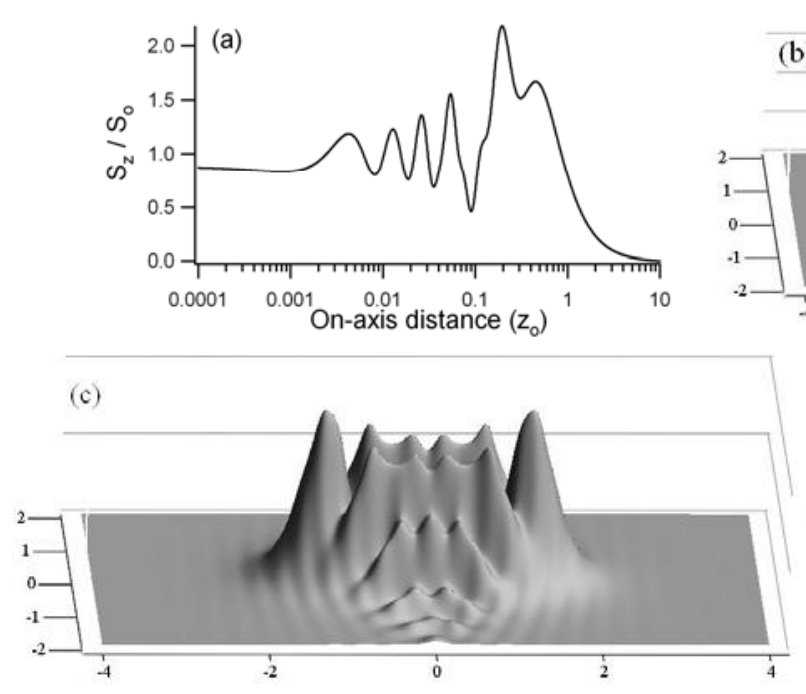
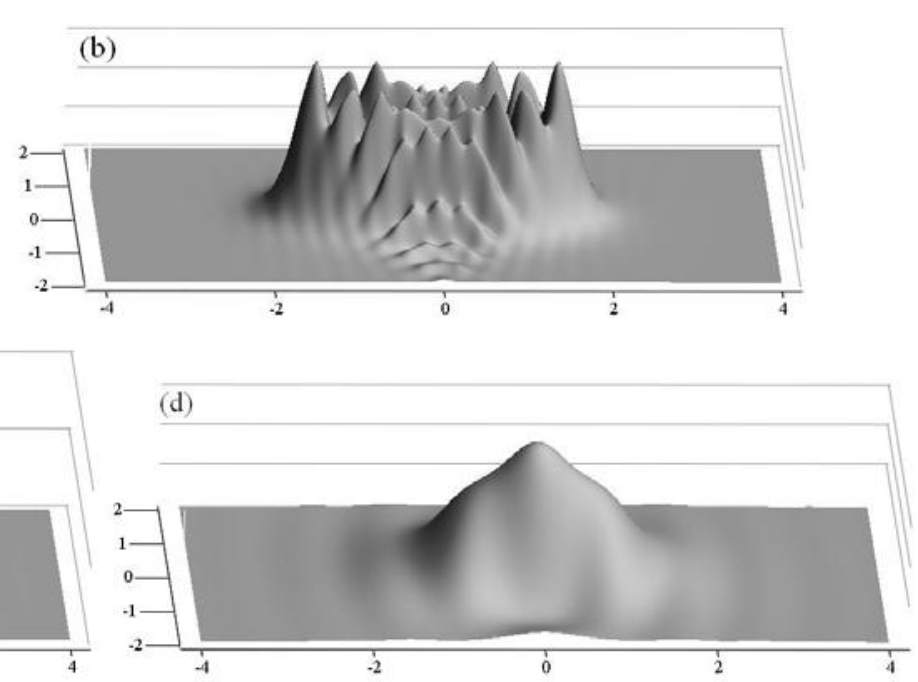

Fig. 4. Calculated intensity of the normalized z-component of the Poynting vector (a) as a function of axial distance from the aperture plane, and beam profiles for (b) an axial distance of $z_{1}=0.054$, (c) $z_{1}=0.09$, and (d) $z_{1}=0.46$, where $z_{1}$ is the on-axis distance normalized to $\mathrm{z}_{0}=k a^{2}$.

\section{CONCLUSIONS}

It has been demonstrated both here and in Ref. [4] that vector diffraction theory using the Hertz vector formalism presents a direct method for calculating all of the electromagnetic field components for plane-wave light fields diffracted by an aperture in an opaque conducting screen. The double integrations over the Hertz vector in the translucent areas of the aperture screen can be performed for any arbitrary aperture shape, or collection of shapes. Computationally efficient single integral forms of the Hertz vector calculation are presented here, and can also be performed with the constraint that the point of interest resides either in the geometrical shadow region or in the geometrically illuminated region. In the aperture plane, the perturbation of the distribution of the light fields is strongly dependent upon the shape of the open aperture area, and its orientation with respect to the polarization of the incident light.

\section{REFERENCES}

1. Allen Wang and Aluizio Prata, Jr., "Lenslet analysis by rigorous vector diffraction theory," J. Opt. Soc. Am. A 12, $1161-1169$ (1995).

2. K. Adam, Y. granik, A. torres, and N. Cobb, "Improved modeling performance with an adapted vectorial formulation of the Hopkins imaging equation," Proc. SPIE 5040, 78-90, (2003).

3. D. G. Flagello, T. Milster, and A. E. Rosenbluth, "Theory of high-na imaging in homogeneous thin films," J. Opt. Soc. Am. A 13, 53 - 64 (1996).

4. Shekhar Guha and Glen D. Gillen, "Description of light propagation through a circular aperture using nonparaxial vector diffraction theory," Opt. Express 13, 1424 - 1447 (2005).

5. A. V. Nesterov and V. G. Niziev, "Vector solution of the diffraction task using the Hertz vector," Phys. Rev. E 71 046608 (2005).

6. M. J. Ehrlich, S. Silver, and G. Held, "Studies of the diffraction of electromagnetic waves by circular apertures and complementary obstacles: the near-zone field," J. Appl. Phys. 26, 336-345 (1955).

7. H. Hertz, Electric Waves, (MacMillan and Co., London, 1893).

8. G. Bekefi, "Diffraction of electromagnetic waves by an aperture in a large screen," J. Appl. Phys. 24, 1123 - 1130 (1953).

9. A. Schoch, "Betrachtungen über das Schallfeld einer Kolbenmembran," Akust. Z. 6, 318 - 326 (1941).

10. A. H. Carter and A. O. Williams, Jr., "A new expansion for the velocity potential of a piston source," J. Accoust. Soc. Am. 23, $179-184$ (1951). 